\title{
ANALYSIS OF DISPLACEMENT OF VIBRATING OF MASS SPRING DUE TO OPPOSITION FORCE
}

\author{
Puskar R. Pokhrel ${ }^{1}$, Bhabani Lamsal ${ }^{2}$, Jeevan Kafle ${ }^{3 *}$, \\ Parameshwari Kattel ${ }^{4}$ \\ ${ }^{1}$ Assistant Professor, Department of Mathematics, Ratna Rajya Laxmi Campus, \\ Kathmandu, TU. \\ ${ }_{2}^{2}$ Lecturer, Sagarmatha Engineering College, Kathmandu, Nepal \\ ${ }^{3}$ Assistant Professor, Central Department of Mathematics, Kathmandu, TU. \\ ${ }^{4}$ Associate Professor, Department of Mathematics, Tri-Chandra Multiple Campus, \\ Kathmandu, TU. \\ *Corresponding author: jeevankafle@yahoo.com
}

\begin{abstract}
Employing the model equation for mass spring system, which is of second order differential equation, we analyze the displacement with various opposition forces applied on the mass of the spring. We observe that if there is no external force on the mass of the spring, the opposition force causes the displacements of mass of spring. We also analyze the system by finding its analytic and numerical solution, and compare their results.
\end{abstract}

Keywords: Mass of spring - opposition forces - analytic and numerical solution.

\section{INTRODUCTION}

Bridges vibrate when heavy vehicles pass along it; the vibration of the wings of the aircraft occurs when there is turbulence. Engineers have a large concern of the vibration of machinery during operations (Zhang et al., 2017). Newton's second law of motion is applied in the transitional systems analysis as the sum of all forces applied to a body equals the product of the vector acceleration of the body times its mass. Shaw et al. (1989) studied the steady state vibrations of a non-linear dynamic vibration absorber. Natsiavas (1992) developed the method of averaging to investigate the stability of non-linear dynamic vibration absorbers. Oueini et al. (1998) presented theoretical and experimental results of the application of the vibration absorber. Oueini et al. (1999) studied the dynamics of a nonlinear 


\section{2}

active vibration absorber. The equations of motion are developed and analyzed through perturbation techniques and numerical simulation. Metallidis and Natsiavas (2000) investigated the dynamics of a mechanical system with continuous characteristics. Zhu et al. (2004) studied the nonlinear dynamics of a two-degree-of-freedom vibration system with nonlinear damping and non-linear springs. Zhou et al. (2020) established the model of the disc spring system in vibration screen.

Generally, mathematical models are used to represent transitional systems which have the basic building of springs, dashpots and masses (Boyce \& DiPrima, 2005; Johnson, 2012). Spring coefficients (stiffness of the spring), dashpot (forces opposing the motion) and mass (inertial or resistance to acceleration) constitute three basic building blocks of mass of spring and their physical representation. The stiffness of a spring is described by the relationship between the forces used to extend or compress a spring, and the resulting extension or compression that arises in the system. The dashpot mainly creates the damping or opposition force that slows down the motion, and it generally consists of a piston moving in a closed cylinder as an idealized representation. According to Newton's second law of motion, bigger the mass is, greater is the force required to give it a specific acceleration (Schilling, 2011; Shin et al., 2018). Both analytic and numerical solution techniques can be employed to solve the problems of vibrating mechanical systems. As some recent advancement in numerical method, Jnawali and Bhatta (2016) developed some variant of Newton type methods based on inverse function. Pokhrel et al. (2020) analyzed the analytic solutions of the model equation of blood flow through artery with mild stenosis, which is in the form of partial differential equation.

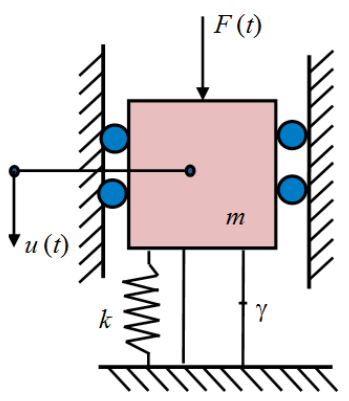

Figure 1: Vibration of mass of string with an external force $F(t)$ (Awrejcewicz, 2012). 
In this paper, we examine the effects of the displacement of mass of spring due to the opposition forces. We observe various displacement patterns of the spring-mass that is also subjected to an opposition force, with and without an external force on the mass of the spring. We analyze the displacements by also finding its numerical solutions using RungeKutta fourth order, and compare the results with analytic solutions. We also analyze the errors on the numerical approximation with respect to the analytic solutions.

\section{MODEL EQUATION}

A mass-spring system which consists of one mass is with single degree of freedom. We take an ordinary spring that resists compression and extension both, and suspend it vertically from fixed support as shown in Figure 1 (Awrejcewicz, 2012). The upper end of the spring is attached to a mass $\mathrm{m}$, and the body is pulled down in a certain distance and then released, then it begins to vibrate. It is assumed that the spring-mass moves strictly vertically. From this physical phenomenon, the model equation is formulated by applying Newton's second law of motion (Boyce and DiPrima, 2005). If there is an external force $F(t)$ act on the body on downward or upward, then the forced vibrations are obtained, and if the constants $F_{0}$, and $\omega_{0}$ are respectively the amplitude and frequency of the force $F(t)$, then $F(t)=F_{0} \cos \omega t$. If the mass is moving downward, the opposition force is directed upward with damping coefficient $\gamma$. So, the following model describes of the motion (Rao, 2000) as:

$m u^{\prime \prime}(t)+\gamma u^{\prime}(t)+k u=F(t)=F_{0} \cos \omega t$.

Here, $t$ denotes the time variable, $m$ is mass, and $k$ is a spring constant of the spring-mass system. The initial conditions are- the initial position $u(0)=\alpha$, and initial velocity $u^{\prime}(0)=\beta$. Along with these initial conditions, the model equation (1) becomes an initial value problem of the spring-mass system. This is a non-homogenous second order ordinary differential equation, so its general solution is $u(t)=u_{\mathrm{c}}(t)+u_{\mathrm{p}}(t)$, where $u_{\mathrm{c}}(t)$ is the transient solution and $u_{\mathrm{p}}(t)$ is steady state solution (Johnson, 2012). If there is no external force on the system, then $F(t)=0$, and the model equation (1) reduces to

$m u^{\prime \prime}(t)+\gamma u^{\prime}(t)+k u=0$.

Let $u(t)=e r t$ be a solution of (2). Then,

$\left(m r^{2}+\gamma r+k\right) e r t=0$. 
Since $e r t \neq 0, \quad m r^{2}+\gamma r+k=0$.

This is quadratic in $r$; the roots of this equation are

$$
r_{1}=\frac{1}{2 m}\left(-\gamma+\sqrt{\gamma^{2}-4 m k}\right), \mathrm{r}_{2}=\frac{1}{2 m}\left(-\gamma-\sqrt{\gamma^{2}-4 m k}\right)
$$

Since $m, \gamma$, and $k$ are positives, $\gamma^{2}-4 k m$ is always less than $\gamma^{2}$. So, the roots $r_{1}$ and $r_{2}$ are distinct and negative if $\gamma^{2}-4 k m>0$. If $\gamma^{2}-4 m k=0$, then the roots $r_{1}$ and $r_{2}$ are real and equal. If $\gamma^{2}-4 m k<0$, then the roots $r_{1}$ and $r_{2}$ are both imaginary with negative real parts. Hence, the three possible solutions of the model (Johnson, 2012) are

$$
u(t)= \begin{cases}\mathrm{c}_{1} e-r_{1} t+\mathrm{c}_{2} e r_{2} t, & \text { if } \gamma^{2}-4 m k>0, \\ e-\gamma t / 2 m\left(\mathrm{c}_{3}+\mathrm{c}_{4} t,\right. & \text { if } \gamma^{2}-4 m k=0, \\ e-\gamma t / 2 m \mathrm{c}_{5} \cos \mu t+\mathrm{c}_{6} \sin \mu t, & \text { if } \gamma^{2}-4 m k<0,\end{cases}
$$

where $\mu=4 m k-\gamma 2 / 2 m$. With initial position $u(0)=\alpha$, and initial velocity, $u^{\prime}(0)=\beta$, the solutions (4) tend to zero as time approaches to infinity, i.e., $u(t)$ dies out as $t$ increases. These analytic solutions are called the transient solution. If an external force $F(t)=F_{0} \cos \omega t$, where $F_{0}$ $>0$ constant, is applied on the mass of the spring, then the steady state solution $u_{\mathrm{p}}(t)=\mathrm{a} \cos \omega t+\mathrm{b} \sin \omega t$, of the model equation (1) does not die out as $t$ increases but keeps on being effective as long as the external force is applied. This solution represents the forced response (Kattel and Kafle, 2018). It is convenient to express $u_{\mathrm{p}}(\mathrm{t})$ as a single trigonometric term rather than as a sum of two terms. Applying the method of undetermined coefficients let the solution be

$u_{\mathrm{p}}(t)=\mathrm{a} \cos \omega t+\mathrm{b} \sin \omega t$,

where $a$ and $b$ are constants of integration. Differentiating (5) with respect to $t$, we get

$u_{\mathrm{p}}{ }^{\prime}(t)=-\mathrm{a} \omega \sin \omega t+\mathrm{b} \omega \cos \omega t, \quad u_{\mathrm{p}}^{\prime \prime}(t)=-\mathrm{a} \omega^{2} \cos \omega t-\mathrm{b} \omega^{2} \sin \omega t$.

Substituting these in (1), we get

-a $m \omega^{2} \cos \omega t-\mathrm{b} m \omega^{2} \sin \omega t-\mathrm{a} \gamma \omega \sin \omega t+\mathrm{b} \gamma \omega \cos \omega t$

$+k \mathrm{a} \cos \omega t+k \mathrm{~b} \sin \omega t=F_{0} \cos \omega t$,

$\Rightarrow\left(\left(k-m \omega^{2}\right) \mathrm{a}+\mathrm{b} \gamma \omega\right) \cos \omega t+\left(-\gamma \omega \mathrm{a}+\left(k-m \omega^{2}\right) \mathrm{b}\right) \sin \omega t=F_{0} \cos \omega t$. 
Equating the coefficients of like terms,

$\left(k-m \omega^{2}\right) \mathrm{a}+\mathrm{b} \gamma \omega=F_{0}$ and $\left(-\gamma \omega \mathrm{a}+\left(k-m \omega^{2}\right) \mathrm{b}\right)=0$.

This forms a system of linear equations for two unknowns a and $b$ with solution:

$$
\mathrm{a}=\frac{\mathrm{F}_{0} \mathrm{~m}\left(\mathrm{k}-\mathrm{m} \omega^{2}\right)}{\left(\mathrm{k}-\mathrm{m} \omega^{2}\right)^{2}+\omega^{2} \gamma^{2}}, \quad \mathrm{~b}=\frac{\mathrm{F}_{0} \omega \gamma}{\mathrm{m}^{2}\left(\mathrm{~m} \omega^{2}\right)^{2}+\omega^{2} \gamma^{2}},
$$

where the expression in the denominator is not equal to zero. Setting $\omega_{0}=$ $\sqrt{\mathrm{k} / \mathrm{m}}$, then

$$
\mathrm{a}=\frac{\mathrm{F}_{0} \mathrm{~m}\left(\omega_{0}^{2}-\omega^{2}\right)}{\mathrm{m}^{2}\left(\omega_{0}^{2}-\omega^{2}\right)^{2}+\omega^{2} \gamma^{2}}, \quad \mathrm{~b}=\frac{\mathrm{F}_{0} \omega \gamma}{\mathrm{m}^{2}\left(\omega_{0}{ }^{2}-\omega^{2}\right)^{2}+\omega^{2} \gamma^{2}} .
$$

Hence, the particular solution of (1) is

$$
u_{\mathrm{p}}(t)=\frac{\mathrm{F}_{0} \mathrm{~m}\left(\omega_{0}{ }^{2}-\omega^{2}\right) \cos \omega \mathrm{t}+\mathrm{F}_{0} \omega \gamma \sin \omega \mathrm{t}}{\mathrm{m}^{2}\left(\omega_{0}{ }^{2}-\omega^{2}\right)^{2}+\omega^{2} \gamma^{2}} .
$$

If we suppose $\mathrm{a}=R \cos \eta, \mathrm{b}=R \sin \eta$, then (3) takes the form $u_{\mathrm{p}}(t)=R$ $\cos \eta \cos \omega t+R \sin \eta \sin \omega t=R \cos (\omega t-\eta)$,

so that $R^{2}=\mathrm{a}^{2}+\mathrm{b}^{2}=\frac{\mathrm{F}_{0}}{\sqrt{\mathrm{m}^{2}\left(\omega_{0}{ }^{2}-\omega^{2}\right)^{2}+\omega^{2} \gamma^{2}}}$, and $\tan \eta=\frac{\mathrm{b}}{\mathrm{a}}=\frac{\omega \gamma}{\mathrm{m}\left(\omega_{0}^{2}-\omega^{2}\right)}$.

In the case of very small damping, this will give satisfactory results over time interval.

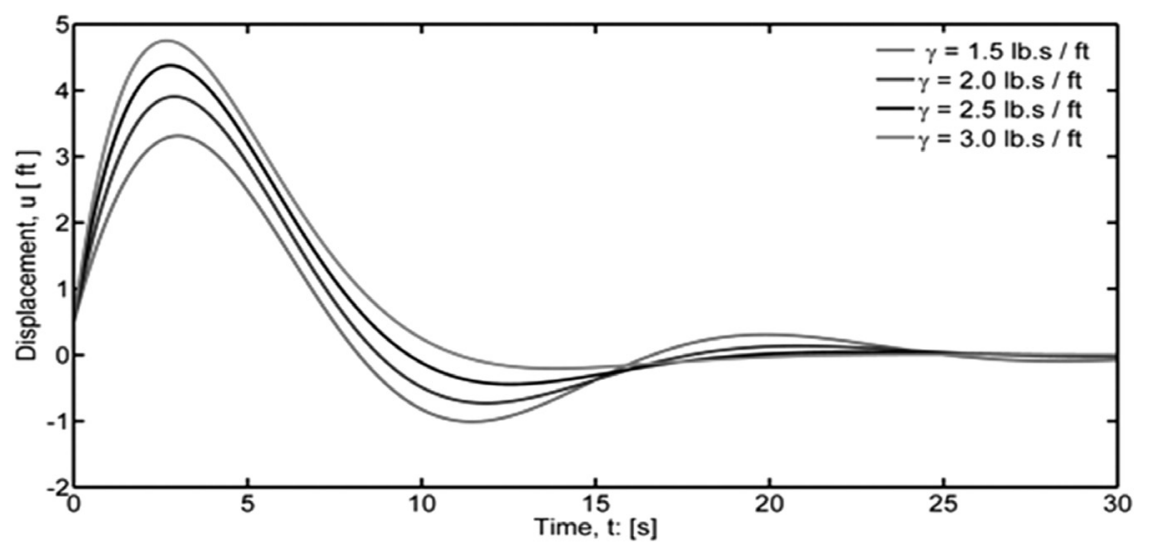

Figure 2: The displacement of mass as function of time with small damping, $\gamma^{2}<4 \mathrm{~m} \mathrm{k}$. 


\section{RESULTS AND DISCUSSION}

With the analytic solutions (4) for the displacement, below, we study the time-behaviour of the displacements for different values of the damping constant $\gamma$. When a mass weighing $6 \mathrm{lb}$ stretches a spring 3 inches, then the spring constant is found as $k=6 \mathrm{lb} / 3 \mathrm{in}=24 \mathrm{lb} / \mathrm{ft}$, and mass is obtained as $m=6 \mathrm{lb} / 32 \mathrm{ft} \mathrm{s}^{2}=3 / 16 \mathrm{lb} \mathrm{s}^{2} / \mathrm{ft}$. In this choice of the parameters, $4 \mathrm{~m} . \mathrm{k}$ $=4.3 / 16.24=18 \mathrm{lb}^{2} . s^{2} / f t^{2}$. To plot the third case $\left(\gamma^{2}<4 \mathrm{mk}\right)$ of the solution for the displacement (4), our $\gamma$ must have value less than $3 \sqrt{ } 2 \mathrm{lb}$. s/ft. Figure 2 reveals this case for the different damping coefficients $\gamma=1.5 \mathrm{lb} . \mathrm{s} / \mathrm{ft}, \gamma=$ $2.0 \mathrm{lb} . \mathrm{s} / \mathrm{ft}, \gamma=2.5 \mathrm{lb} . \mathrm{s} / \mathrm{ft}, \quad \gamma=3.0 \mathrm{lb} . \mathrm{s} / \mathrm{ft}$, the displacement are 3.3113 $f t, 3.9071 \mathrm{ft}, 4.3761 \mathrm{ft}, 4.7521 \mathrm{ft}$ at time

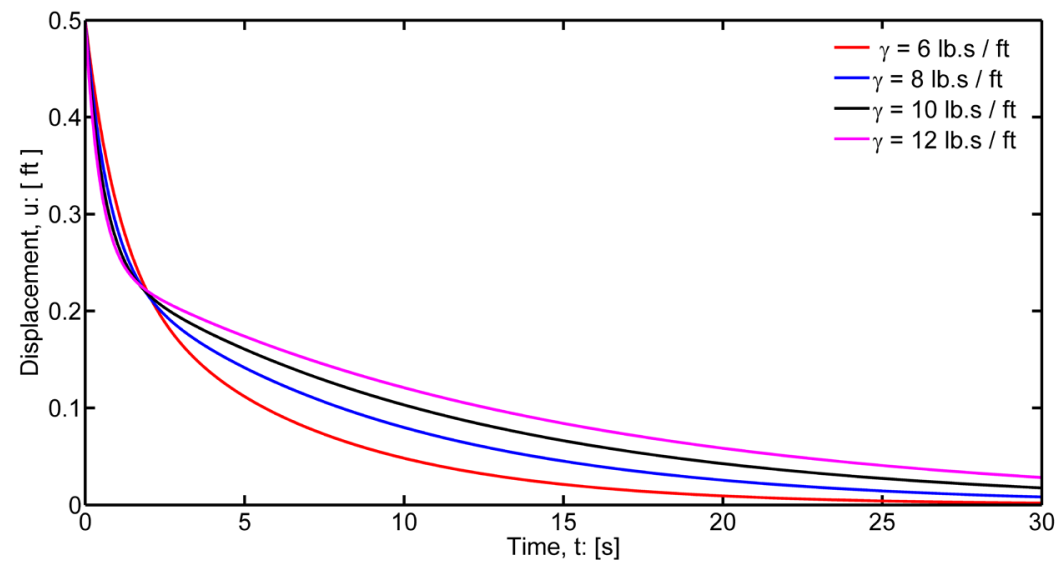

Figure 3: The displacement of mass as function of time with larger damping, $\gamma^{2}>4 \mathrm{~m} \mathrm{k}$.

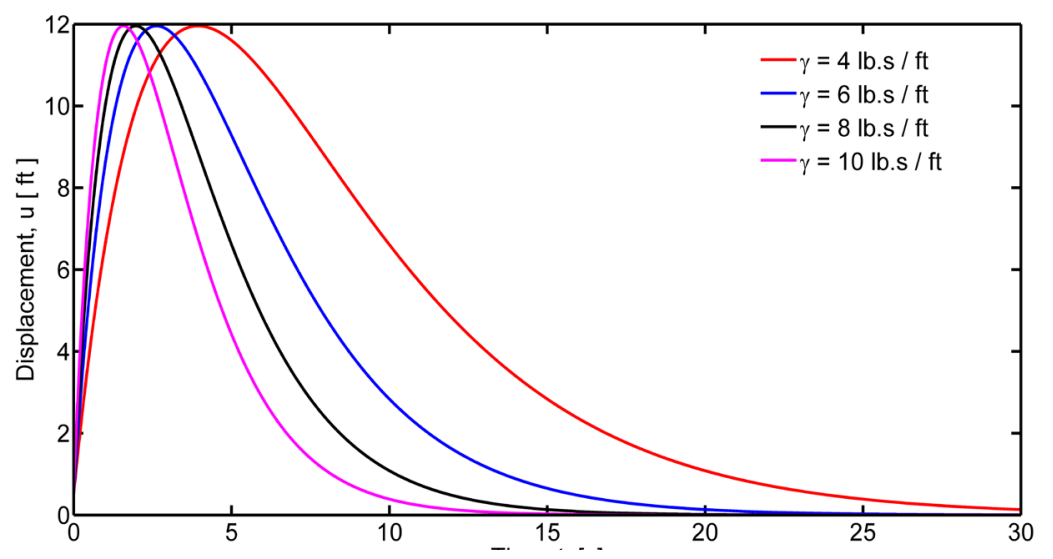

Figure 4: The displacement of mass as function of time with the case, $\gamma^{2}$ $=4 \mathrm{~m} \mathrm{k}$. 
$t=2.201 \mathrm{~s}$, and with the initial displacement of mass-spring, $u(0)$ $=1 / 2 \mathrm{ft}$, and initial velocity $u^{\prime}(0)=0 \mathrm{ft} \mathrm{s} \mathrm{s}^{-1}$. In the case of $\gamma^{2}<4 k m$, the opposition forces affect the displacement of the spring differently, whereas there is almost no displacement as $t$ approaches $30 \mathrm{~s}$.

With the same spring constant $k=24 \mathrm{lb} / \mathrm{ft}$, mass $m=3 / 16 \mathrm{lb} . \mathrm{s}^{2} /$ $f t$, and the initial position of the mass of spring, $u(0)=1 / 2 \mathrm{ft}$, and initial velocity $u^{\prime}(0)=0 f t s^{-1}$, but with the different case of $\gamma^{2}>4 k m$ (i.e., $\gamma>3 \sqrt{ } 2$ $l b . s / f t$ ), the displacements (almost) vertically drop to reach $u=0.2134 \mathrm{ft}$ for the increasing damping coefficients $\gamma=6 \mathrm{lb} . \mathrm{s} / \mathrm{ft}, \gamma=8 \mathrm{lb} . \mathrm{s} / \mathrm{ft}, \gamma=10 \mathrm{lb}$. $s / f t, \gamma=12 \mathrm{lb} . s / f t$ at time $t=2.21 \mathrm{~s}$ as shown in Figure 3. The displacement vanishes at time $t=10 \mathrm{~s}$ for damping coefficient $\gamma=6 \mathrm{lb} . \mathrm{s} / \mathrm{ft}$, whereas for damping coefficient $\gamma=8 \mathrm{lb} . \mathrm{s} / \mathrm{ft}$, the displacement vanishes at time $t=$ $25 s$. When opposition force increases, the displacements take longer time to vanish. This is physically meaningful.

Figure 4 plots the solution for the displacement of the vibrating system of the second case of (4), where $\gamma^{2}=4 k \mathrm{~m}$. So, we study the different behaviours of the displacements of the system, we choose fixed $m=1 / 8$ $l b . s^{2} / f t$, but with different spring constants, $k=32,72.128$ and $200 \mathrm{lb} /$ $\mathrm{ft}$, with the respective damping forces $\gamma=4,6,8$ and $10 \mathrm{lb} . \mathrm{s} / \mathrm{ft}$. In each case, the amplitude (maximum value) of the displacement is of $12 \mathrm{~m}$, but they are attained in longer time for smaller opposition forces. It is equally interesting to observe that the displacement dies out earlier for larger, and later for smaller opposition forces.

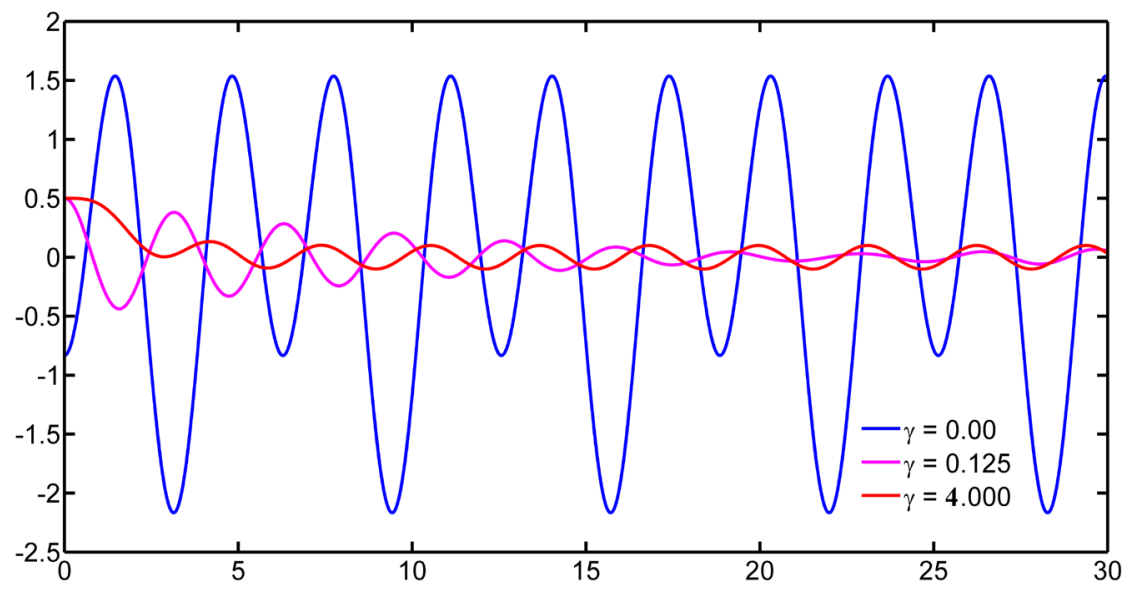

Figure 5: The displacement of mass as function of time with external force $\mathrm{F}=\mathrm{F}_{0} \cos \omega \mathrm{t}$. 
Previous analyses (Figure 2-4) for displacements were for the vibrating system with opposition forces, but without the external force. Our next analysis (Figure 5) is for the system with an external force $F=$ $F_{0} \cos \omega t$, as defined earlier. With the solutions with (4) together with for the particular integral (5), here, we reveal the behavior of displacements without the opposition force $(\gamma=0 \mathrm{lb} s / f t)$ and with other two opposition forces $(\gamma=0,0.125,4 \mathrm{lb} . s / f t)$. In case if there is no opposition force, (i.e., $\gamma=0$ ), Figure 5 reveals that the displacement is up to $u=1.5 \mathrm{ft}$ in the time interval $0 \leq t \leq 30 \mathrm{~s}$. In either of the cases $\gamma^{2}<4 \mathrm{~km}$ or $\gamma^{2}>4 \mathrm{~km}$, the displacement converges to zero after $t=30 \mathrm{~s}$ for $\gamma=0.125 \mathrm{lb}$. s $/ \mathrm{ft}$ and $\gamma=$ $4 \mathrm{lb} . s / f t$ as in Figure 5.

\section{NUMERICAL SOLUTION}

Here, we also solve the model equation numerically, and maximum iterations processes are employed to make less error in the numerical solution (Jnawali and Bhatta, 2016; Pokhrel, 2017 ). Consider the time interval $T=\left\{t: 0<t<t_{\mathrm{N}}\right\}$, and it is subdivided into the $(N+1)$ equal subinterval with time step $\Delta t=h$ whose ends points are the mesh points $t_{\mathrm{i}}=0+\mathrm{i} h$, for $\mathrm{i}=0,1, \ldots N+1$, where $h=\left(t_{\mathrm{N}}-0\right) /(N+1)$. Introducing the variable $z=z(t)$ (Kreyszig, 1979 ), the model equation (1) can be written in the form:

$$
\begin{aligned}
& \frac{d u}{d t}=z=f_{1}(t, u, z) \text { with } u(0)=\frac{1}{2} \\
& \frac{d u}{d t}=\frac{F_{0}}{m} \cos \omega t-\frac{\gamma}{m} z-\frac{k}{m} u=f_{2}(t, u, z) \text { with } z(0)=u^{\prime}(0)=0 .
\end{aligned}
$$

Applying Runge-Kutta fourth order method, it is written ( Kreyszig, 1979; Pokhrel, 2018) as

$u_{\mathrm{i}+1}=u_{\mathrm{i}}+\frac{1}{6}\left(k_{1}+2 k_{2}+2 k_{3}+k_{4}\right)$, and $z_{\mathrm{i}+1}=z_{\mathrm{i}}+\frac{1}{6}\left(l_{1}+2 l_{2}+2 l_{3}+l_{4}\right)$; where $k_{1}=h f_{1}\left(t_{\mathrm{i}}, u_{\mathrm{i}}, z_{\mathrm{i}}\right)=h z_{\mathrm{i}}, l_{1}=h f_{2}\left(t_{\mathrm{i}}, u_{\mathrm{i}}, z_{\mathrm{i}}\right)=h\left(\frac{F_{0}}{m} \cos \omega t_{i}-\frac{\gamma}{m} z_{i}-\frac{k}{m} u_{i}\right)$

$$
\begin{aligned}
& k_{2}=h f_{1}\left(t_{\mathrm{i}}+0.5 h, u_{\mathrm{i}}+0.5 k_{1}, z_{\mathrm{i}}+0.5 l_{1}\right)=h\left(z_{\mathrm{i}}+0.5 h\right) \\
& l_{2}=h f_{2}\left(t_{\mathrm{I}}+0.5 h, u_{\mathrm{I}}+0.5 k_{1}, z_{\mathrm{i}}+0.5 l_{1}\right) \\
& =h\left(\frac{F_{0}}{m} \cos \omega\left(t_{i}+0.5 h\right)-\frac{\gamma}{m}\left(z_{i}+0.5 l_{i}\right)-\frac{k}{m}\left(u_{i}+0.5 k_{i}\right)\right) \\
& k_{3}=h f_{1}\left(t_{\mathrm{i}}+0.5 h, u_{\mathrm{i}}+0.5 k_{2}, z_{\mathrm{i}}+0.5 l_{2}\right)=h\left(z_{\mathrm{i}}+0.5 h\right)
\end{aligned}
$$




$$
\begin{aligned}
& l_{3}=h f_{2}\left(t_{\mathrm{i}}+0.5 h, u_{\mathrm{i}}+0.5 k_{2}, z_{\mathrm{i}}+0.5 l_{2}\right) \\
& =h\left(\frac{F_{0}}{m} \cos \omega\left(t_{i}+0.5 h\right)-\frac{\gamma}{m}\left(z_{i}+0.5 l_{2}\right)-\frac{k}{m}\left(u_{i}+0.5 k_{2}\right)\right) \\
& k_{4}=h f_{1}\left(t_{\mathrm{i}}+h, u_{\mathrm{i}}+k_{3}, z_{\mathrm{i}}+l_{3}\right)=h\left(z_{\mathrm{i}}+h\right) \\
& l_{4}=h f_{2}\left(t_{\mathrm{i}}+h, u_{\mathrm{i}}+k_{3}, z_{\mathrm{i}}+l_{3}\right) \\
& =h\left(\frac{F_{0}}{m} \cos \omega\left(t_{i}+0.5 h\right)-\frac{\gamma}{m}\left(z_{i}+0.5 l_{3}\right)-\frac{k}{m}\left(u_{i}+0.5 k_{3}\right)\right), \text { for } \mathrm{i}=1,2, \ldots, \mathrm{N} .
\end{aligned}
$$

Table 1: The error analysis of the displacement of a spring-mass system.

\begin{tabular}{cccc}
\hline Time: $\boldsymbol{t}[\mathbf{s}]$ & $\begin{array}{c}\text { Disp.: } \boldsymbol{u}[\mathbf{f t}] \\
\text { Numerical }\end{array}$ & $\begin{array}{c}\text { Disp.: } \boldsymbol{u} \text { [ft] } \\
\text { Analytical }\end{array}$ & Error $(\varepsilon)$ \\
\hline 0.00 & 0.5000 & 0.5 & 0 \\
3.75 & 0.1130 & -0.5264 & 0.0808 \\
7.50 & -0.4456 & 0.3086 & 0.0926 \\
11.25 & -0.0729 & -0.3335 & 0.1153 \\
15.00 & 0.2160 & 0.1828 & 0.1253 \\
18.75 & 0.1969 & -0.1734 & 0.0957 \\
22.50 & -0.2182 & 0.1133 & 0.1146 \\
26.25 & -0.0977 & -0.0511 & 0.0535 \\
30.00 & 0.0575 & 0.0813 & 0.0835 \\
33.75 & 0.1609 & 0.0309 & 0.0145 \\
37.50 & -0.0777 & 0.066 & 0.0474 \\
41.25 & -0.0793 & 0.0728 & 0.0089 \\
45.00 & -0.0013 & 0.0511 & 0.0143 \\
48.75 & 0.0887 & 0.0784 & 0.0148 \\
52.50 & 0.0024 & 0.0281 & 0.0121 \\
56.25 & -0.0635 & 0.0561 & 0.0086 \\
60.00 & -0.0022 & -0.0035 & 0.0304 \\
\hline & & & \\
\hline & & & 0 \\
\hline
\end{tabular}

Note: The numerical and analytical results from the model equations. 


\section{NUMERICAL RESULTS}

Figure 6 describes the numerical solution (o) and the analytic solution (solid line) in time interval $0 \leq t \leq 60 \mathrm{~s}$. The initial position of a mass of spring with $m=1 \mathrm{lb} \mathrm{s}^{2} / \mathrm{ft}$ is $u(0)=1 / 2 \mathrm{ft}$, initial velocity zero, spring coefficient $k=1 \mathrm{lb} / \mathrm{ft}$. When opposition force is applied with damping coefficient $\gamma=6 \mathrm{lb} \mathrm{s} / \mathrm{ft}$, the spring is displaced with a sharp oscillation in the spring.

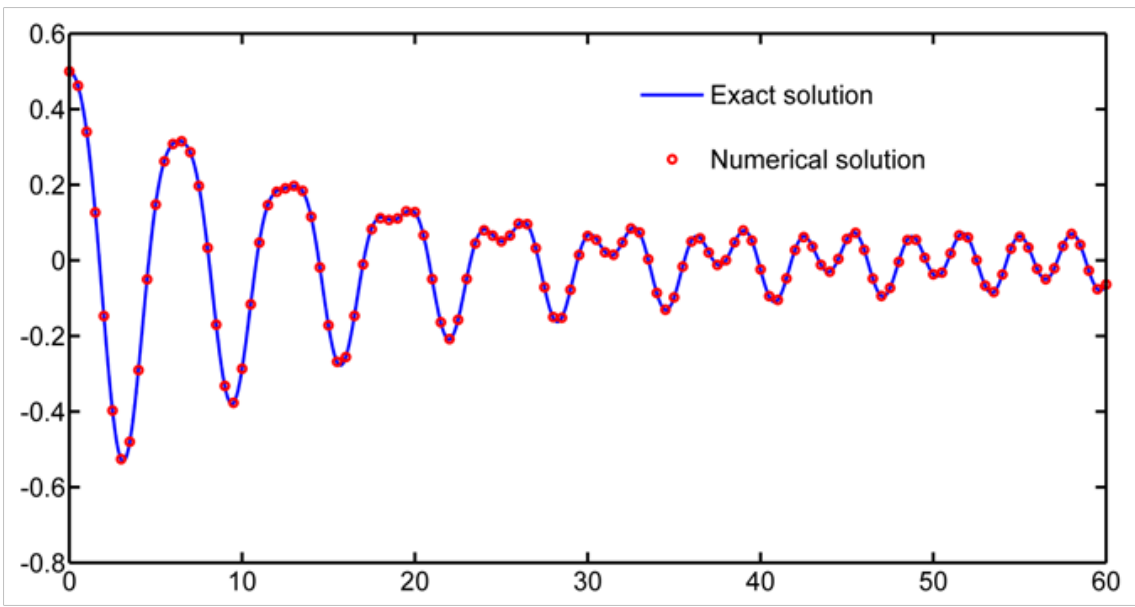

Figure 6: The displacement of mass with external force $F=F_{0} \cos \omega t$ obtained from both numerical and analytical approaches.

Then after it, there is damping in the oscillation. The damping continues with substantial decrease in the amplitude of the displacement as shown in Figure 6. The analytical and numerical solutions are almost in line with each other. The errors in analytical and numerical solutions are shown in Table 1.

\section{CONCLUSION}

We presented the model equation and the analytic solution of mass of spring with external and viscous (damping or opposition) force. We examined the displacement of mass-spring system with various damping coefficients. We found that higher the damping coefficients, higher are the displacement of mass of spring in most cases of the analytic solutions. We analyzed the displacement of mass of spring by applying and without applying an external force. We also used Runge-Kutta fourth order method to solve the model equation numerically. We found that the numerical 
and analytical solutions are almost same. We also analyzed the error between the analytic and numerical solution. Error analysis showed that the displacement of the mass of spring due to opposition forces can be integrated numerically as well.

\section{REFERENCES}

Awrejcewicz, J. (2012). Classical mechanics: Dynamics, Springer, New York.

Boyce, W.E., \& DiPrima, R.C.(2005). Elementary differential equations and boundary value problems, John Wiley \& Sons, Inc.

Jha, T. (2018). Velocity Detection from a motion blur image using radon transformation. Tribhuvan University Journal, 32(2): 243-248.

Jnawali, J., \& Bhatta, C. (2016). Iterative methods for solving nonlinear equations with fourth-order convergence. Tribhuvan University Journal, 30(2): 65-72.

Johnson, R.S. (2012). Second order Ordinary Differential Equation, Ventus Publshing ApS.

Kattel, P., Kafle, J. (2018). Differential equations with applications, Sukunda Pustak Bhawan, Kathmandu

Kreyszig, E. (1979). Advanced engineering mathematics, John Wiley \& Sons, Inc, New York.

Metallidis, P., \& Natsiavas, S. (2000). Vibration of a continuous system with clearance and motion constraints, International Journal of Non-linear Mechanics, 35(4): 675-690.

Natsiavas, S.(1992). Steady state oscillations and stability of non-linear dynamic vibration absorbers, Journal of Sound and Vibration, 156 (2): $227-245$.

Oueini, S.S., Nayfeh, A.H. \& Pratt, J.R. (1998). A nonlinear vibration absorber for flexible structures, Nonlinear Dynamics, 15: 259 -282.

Oueini, S.S., Chin, C.M. \& Nayfeh, A.H.(1999). Dynamics of a cubic nonlinear vibration absorber, Nonlinear Dynamics, 20: 283-295.

Pokhrel, J.K. (2017). A Study on maxima and minima for single real valued function. Tribhuvan University Journal, 31(1-2): 45-50.

Pokhrel, P.R. (2018). Numerical methods for engineering, Vidharthy Pustak Bhandar, Kathmandu, Nepal. 
Pokhrel, P.R., Kafle. J., Kattel, P. \& Gaire, H.P. (2020) Analysis of blood flow through artery with mild stenosis. Journal of Institute of Science \& Technology, 25(2): 33-38, doi: org/10.3126/jist. v25i2.33732.

Rao, S.S. (2000). Mechanical vibrations, Addsion-Wesley Publishing Company, New York.

Schilling, R.J. (2011). Applied numerical methods for engineering, Cengage Learning India Private Limited, Deilhi-110092, India.

Shaw, J., Shaw, S.W. \& Haddow, A.G. (1989). On the response of the non-linear vibration absorber, International Journal of Non-linear Mechanics, 24(4): 281-293.

Shin, J.-H., Talib, E. \& Kwak, M.K. (2018). Vibration of a string against multiple spring-mass-damper stoppers, Journal of Sound and Vibration, 414: 157-173.

Zhang, X., Wen, B. \& Zhao C. (2017). Vibratory synchronization transmission of a cylindrical roller in a vibrating mechanical system excited by two exciters, Mechanical Systems and Signal Processing, 96: 88-103.

Zhou, J., Zhang, C., Wang, Z. \& Mao, K. (2020). Study on dynamic characteristics of the disc spring system in vibration screen, Hindawi Shock and Vibration, 2020. doi.org/10.1155/2020/3518037.

Zhu, S. J., Zheng, Y. F. \& Fu, Y. M. (2004). Analysis of non-linear dynamics of a two-degree-of-freedom vibration system with non-linear damping and non-linear spring, Journal of Sound and Vibration, 271(1-2): 15-24. 